UNIVERSITE DU QUEBEC

MEMOIRE PRESENTE

A

LUNIVERSITE DU QUEBEC A TROIS-RIVIERES

COMME EXIGENCE PARTIELLE

DE LA MAITRISE EN PSYCHOLOGIE

\begin{abstract}
PAR
DANIS PAGEAU

STRUCTURATION EDUCATIVE PARENTALE ET FONCTIONNEMENT COGNITIF DES JEUNES ENFANTS
\end{abstract}

SEPTEMBRE 1992 
Université du Québec à Trois-Rivières

Service de la bibliothèque

\section{Avertissement}

L'auteur de ce mémoire ou de cette thèse a autorisé l'Université du Québec à Trois-Rivières à diffuser, à des fins non lucratives, une copie de son mémoire ou de sa thèse.

Cette diffusion n'entraîne pas une renonciation de la part de l'auteur à ses droits de propriété intellectuelle, incluant le droit d'auteur, sur ce mémoire ou cette thèse. Notamment, la reproduction ou la publication de la totalité ou d'une partie importante de ce mémoire ou de cette thèse requiert son autorisation. 
Ce document est rédigé sous la forme d'un article scientifique, tel qu'il est stipulé dans les règlements des études avancées (art. 16.4) de l'Université du Québec à Trois-Rivières. L'article a été rédigé selon les normes de publication d'une revue reconnue et approuvée par le Comité d'études avancées en psychologie. Le nom du directeur de recherche pourrait donc apparaître comme co-auteur de l'article soumis pour publication. 
Table des matières

INTRODUCTION

PROBLEMATIQUE.

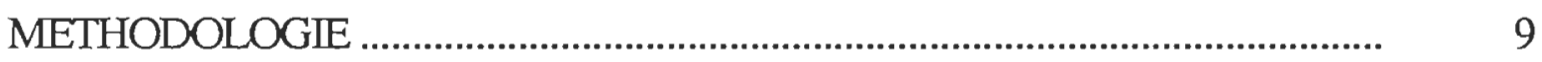

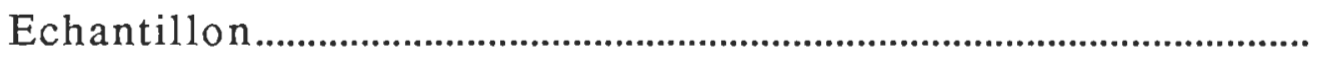

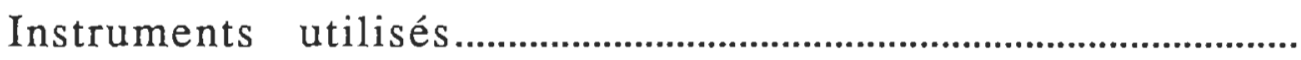

L'expérience

RESULTATS

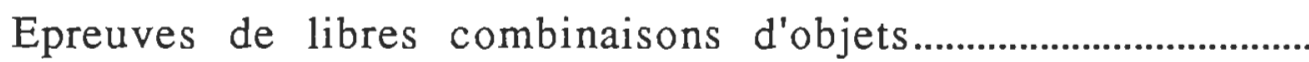

Structuration éducative parentale.

La relation entre la structuration éducative parentale et le développement cognitif de l'enfant.

REFERENCES 


\title{
Structuration éducative parentale et fonctionnement cognitif des jeunes enfants.
}

\author{
Danis Pageau et Ercilia Palacio-Quintin \\ Groupe de recherche en développement de l'enfant \\ Université du Québec à Trois-Rivières, Québec, Canada
}

\section{INTRODUCTION}

Plusieurs auteurs ont montré qu'il existe, dès l'âge préscolaire, une relation entre le statut socio-économique du parent et le développement intellectuel des enfants. En effet, les enfants provenant de milieux plus aisés présentent un niveau intellectuel plus élevé (Golden, Birns, Bridger et Moss, 1971; Goldstein, Meyer et Egeland, 1978; Palacio-Quintin et Jourdan-Ionescu, 1991; Scarr, 1981). Certains auteurs, afin d'expliquer cette relation, se sont questionnés sur les variables qui sont effectivement responsables des différences existant entre les enfants provenant de milieux socio-économiques différents. Nous pouvons regrouper l'ensemble de ces travaux en deux catégories, selon le type de variables de l'environnement qui sont prises en considération.

D'un côté, des recherches ont porté sur les caractéristiques de l'environnement qui favorisent le développement intellectuel des enfants. Les travaux de Bradley et Caldwell ont particulièrement marqué ce domaine de recherche (Bradley et Caldwell, 1976a, 1976b, 1977, 1978; Caldwell et Richmond, 1967, Caldwell, Heider et Kaplan, 1966; Elardo, Bradley et Caldwell, 1975). L'échelle HOME ("Home Observation for the Measurement of Environment") qu'ils ont créée mesure la présence dans l'environnement de caractéristiques variées considérées comme étant favorables au développement de l'intelligence. De nombreuses recherches ont été réalisées, par la suite, à l'aide de l'échelle HOME, afin de démontrer l'effet de certaines variables de l'environnement familial sur le développement des enfants (Bee, Barnard, Eyres, Gray, Hammond, Spietz, Snyder et Clark, 1982; Gottfried, 1984; Palacio-Quintin et Jourdan-Ionescu, 1991). Un 
autre instrument, le Purdue Home Stimulation Inventory (PHSI) a été élaboré et utilisé par Wachs $(1978,1979)$ dans le cadre de recherches similaires.

D'autres auteurs ont plutôt cherché l'explication des différences que l'on retrouve entre les enfants provenant de milieux socio-économiques différents, en étudiant les comportements éducatifs des parents. Notre recherche s'inscrit dans ce deuxième courant qu'est l'étude des attitudes et pratiques éducatives et de leur influence sur le développement intellectuel des enfants.

\section{CONTEXTE THEORIQUE}

Différents aspects des attitudes parentales ont été mis en relation avec l'intelligence des enfants.

Citons d'abord deux recherches longitudinales d'envergure devenues classiques. La Fels $\underline{\text { Research (Baldwin, Kalhorn et Breese, 1945; Baker, Nelson et Sontag, 1958) et la Berkeley }}$ Growth Study (Bayley, 1933). Chacune de ces deux recherches a fourni, entre autre, des données relatives aux attitudes parentales et à leurs liens avec le développement intellectuel des enfants.

Baldwin et al. (1945), membres du groupe de la Fels Research, regroupent les différentes attitudes parentales en trois catégories: acceptation de l'enfant, protection ${ }^{1}$ et démocratie à la maison. Etudiant la relation entre ces attitudes et le niveau intellectuel, ils trouvent que l'enseignement démocratique est plus favorable au développement intellectuel et que les types protecteurs et restrictifs (pôle négatif de l'attitude démocratique) seraient moins favorables. Plus tard, Baker et al (1958), reprenant les données de la Fels Research ${ }^{2}$, utilisent un regroupement différent des variables (affection, protection, contrôle coopératif, contrôle coercitif, harmonie familiale et accélération maternelle ${ }^{3}$ ). Ils s'intéressent ensuite aux QI des enfants, selon qu'ils augmentent ou diminuent dans le temps (i.e. entre deux évaluations consécutives), et vérifient la

1 Ceci est le terme utilisé par Lautrey (1980) pour traduire l'expression anglaise "indulgence" utilisée par les auteurs. Ce terme apparaît plus adéquat, selon la définition donnée par Baldwin elt al., que la traduction littérale indulgence.

2 La recherche de Baker et all. a été faite sur un plus grand nombre de sujets que celle de Baldwin et al. (1945).

3 L'accélération matemelle se définit par la tendance de la mère à se montrer excessivement impliquée dans le développement des habiletés motrices et cognitives de l'enfant (Lautrey, 1980). 
relation entre les attitudes des parents et l'augmentation ou la diminution du QI. Ils trouvent que le contrôle coopératif et l'accélération maternelle sont en relation statistiquement significative avec un QI ascendant chez des enfants de 8 ans 6 mois à dix ans. Aucune relation significative n'est trouvée entre attitudes des parents et variation du QI pour leur groupe d'enfants évalués à 4 ans 6 mois et 6 ans. Moss et Kagan (1958) mesurent d'une façon différente l'attitude accélératrice de la mère et la mettent en relation avec le développement intellectuel des enfants de trois et six ans, toujours à partir de l'échantillon de la Fels Research. La seule relation significative trouvée concerne les garçons de trois ans.

Pour leur part, Becker, Peterson, Hellmer, Shoemaker et Quay (1959) trouvent que, autant pour le père que pour la mère, l'attitude protectrice est corrélée avec un bas QI chez l'enfant et que l'attitude démocratique de la mère est corrélée avec un haut QI de l'enfant.

Les travaux de la Berkeley Growth Study ont permis l'examen des corrélations entre 18 variables relatives aux pratiques éducatives. Une analyse factorielle a permis de dégager les dimensions suivantes: Amour/Hostilité et Autonomie/Contrôle (Schaefer, 1959). Les principaux résultats trouvés par Bayley et Schaefer (1964), lorsqu'ils étudient la relation entre ces dimensions et le QI sont difficiles à interpréter. En effet, ils trouvent que, jusqu'à un an, le QI des garçons est corrélé positivement avec les comportements hostiles de la mère et négativement avec les comportements acceptants de celle-ci, mais, de 4 ans à l'âge adulte, le QI des garçons corrèle positivement avec l'affection de la mère et négativement avec l'hostilité. Chez les filles, de la première année jusqu'à l'âge de 4 ans, il existe une relation positive entre le QI et la dimension affection et une relation négative entre le QI et la dimension hostilité, mais, chez les filles de plus de 4 ans, aucune relation n'est trouvée entre les pratiques éducatives et le QI.

Outre les résultats issus de ces deux recherches importantes (la Fels Research et la Berkeley Growth Study), d'autres chercheurs se sont arrêtés à l'étude des attitudes parentales et du facteur "contrôle".

Certains auteurs présentent des résultats de recherches qui vont dans le même sens que ceux issus de la Fels Research et selon lesquels l'attitude démocratique serait favorable au développement intellectuel. Ainsi, Hurley (1959) montre que les enfants ayant les QI les plus 
élévés ont des parents moins autoritaires et moins restrictifs que les autres. Cross (1966), s'intéressant au niveau conceptuel des enfants, montre que ceux qui ont un plus haut niveau conceptuel (évalué avec une tâche de complètement de phrase) proviennent de familles démocratiques et ceux qui ont un niveau conceptuel plus bas proviennent de familles autocratiques ${ }^{4}$. Pour sa part Goldschmid (1968) ne trouve pas de relation entre l'attitude dominante de la mère et le QI de l'enfant mais trouve une corrélation négative avec la réussite de l'enfant aux épreuves de conservation. Les résultats que présente Nichols (1964) montrent que l'attitude contrôlante de la mère est corrélée négativement avec la créativité des enfants. Banner (1979) trouve que les mères qui sont restrictives, dominantes, intrusives et intolérantes avec leurs fils ou dominantes, protectrices, autoritaires et directives avec leurs filles ont des enfants qui réussissent moins bien académiquement. Enfin, Busse (1969) montre que les attitudes de commandement de la mère et l'expression de normes rigides chez le père corrèlent négativement avec une flexibilité de la pensée chez l'enfant.

Par contre, Drews et Teahan (1957) trouvent, en contrôlant le QI, que les mères dont les enfants réussissent le mieux en classe sont plus autoritaires et plus restrictives. Les résultats de Kent et Davis (1957) vont dans le même sens. Ils trouvent, en effet, que les enfants ayant les QI les plus élevés sont ceux dont les parents exigent de l'enfant qu'il se conforme à un modèle idéal inflexible, ne tolèrent aucun écart et sont capables de subordonner leur affection et leur acceptation à sa réussite. Pour sa part, Bing (1963) montre que les enfants qui ont des mères exigeantes réussissent mieux dans les épreuves verbales, alors que les enfants qui réussissent mieux dans les épreuves numériques et spatiales sont ceux à qui les mères laissent plus de liberté pour expérimenter eux-mêmes.

Plusieurs des résultats présentés semblent contradictoires. Nous pensons que cela peut s'expliquer, en partie, par la non différenciation faite entre "stimulation du comportement académique" et "orientation parentale vers la performance". En effet, Storfer (1990) montre que la stimulation du comportement académique de la part des parents est corrélée avec le QI des enfants,

4 Cross utilise plutôt les termes de familles interdépendantes et unilatérales mais la définition qu'il en donne nous permet de les rapprocher des concepts d'attitudes démocratiques et autocratiques. 
alors que l'orientation parentale vers la performance ne l'est pas. De plus, on ne trouve pas de relation entre ces deux attitudes. Storfer en conclut que le simple fait d'encourager l'enfant à performer n'a pas les effets escomptés sur le développement intellectuel si cette attitude n'est pas accompagnée d'une stimulation culturelle et académique.

D'autres recherches se sont plutôt intéressées à la dimension "amour-hostilité" dans les attitudes parentales. Ainsi, Radin (1974) montre que, chez les enfants de 4 ans, la chaleur affective maternelle est liée au QI des filles mais non pas à celui des garçons. Elle montre également une relation entre la chaleur affective du père et le QI des garçons de 4 ans. Les résultats de Hamilton (1971) vont dans le même sens. Il trouve, en effet, que les enfants dont la mère a une attitude acceptante réussissent mieux dans une tâche de conservation que ceux dont la mère est rejetante. Aussi, Hilliard et Roth (1969) montrent que l'attitude acceptante de la mère est reliée à la réussite académique des enfants. Wulbert, Inglis, Kriegsmann et Mills (1975) s'intéressent à la relation entre le type d'interaction mère-enfant et la réussite aux épreuves verbales. Dans le groupe des enfants sans retard verbal, ils observent que les mères leurs parlent plus souvent d'une manière acceptante et chaleureuse, essaient de les raisonner et disent passer plus de temps à jouer avec leurs enfants et à les encourager à apprendre. Rappelons enfin les résultats issus de la BGS présentés par Bayley et Schaefer (1964), que nous avons cités plus haut, selon lesquels l'attitude acceptante de la mère est reliée positivement au QI des garçons de plus d'un an et à celui des filles de 4 ans et moins.

\section{PROBLEMATIQUE}

Les mesures d'attitudes parentales et d'intelligence utilisées par les différents auteurs sont extrêmement variées et des appellations différentes sont souvent utilisées pour désigner des réalités très proches. Ainsi, les termes démocratie et contrôle coopératif semblent désigner des réalités très similaires et sont opposés à l'attitude autoritaire, dominante, restrictive, contrôlante ainsi qu'à l'expression de normes rigides. De même, les termes acceptation, chaleur affective, harmonie familiale et affection sont autant de termes désignant une réalité commune. En ce qui concerne 
l'intelligence des enfants, on mesure le quotient intellectuel, le fonctionnement opératoire, le niveau conceptuel, la créativité, la flexibilité de la pensée, le rendement dans des tâches verbales ou numériques, la réussite scolaire, etc. De plus, les résultats obtenus par les chercheurs sont souvent différents et parfois contradictoires.

Devant la difficulté d'unifier les recherches et devant l'incohérence des résultats obtenus, Lautrey (1980) remet en question le choix des variables. Il constate que les auteurs choisissent souvent les variables à mesurer en fonction de leur expérience et/ou à partir de leur intuition. Il critique également la démarche très répandue qui consiste à regrouper les variables qui varient en même temps et démontre l'inadéquacité de cette méthode dans une recherche à visée explicative. Enfin, il suggère que dans une recherche où on vise à mettre en lumière l'effet de telle pratique éducative sur tel aspect du développement, les variables choisies doivent être tirées d'un cadre théorique approprié. Lautrey (1980) s'inspire donc de la théorie du développement cognitif de Piaget pour étudier la relation entre les attitudes parentales et l'intelligence des enfants.

A partir du processus d'équilibration proposé par Piaget, Lautrey suggère que deux caractéristiques environnementales sont favorables au développement cognitif: 1) être source de perturbations, i.e. de résistances aux schèmes d'assimilation du sujet; 2) offrir les conditions nécessaires aux rééquilibrations, donc aux constructions. Eliminant la possibilité que dans un environnement les deux caractéristiques soient absentes à la fois, il retient trois types de milieu familial: 1) le milięu familial faiblement structuré (événements imprévisibles et irréguliers) qui ne répond qu'à la première des deux caractéristiques; 2 ) le milieu rigide, répondant à la deuxième caractéristique, à cause de la régularité avec laquelle se produisent les événements; 3) le milieu à structuration souple, offrant à l'enfant les deux caractéristiques en même temps, étant donné que les événements qui s'y produisent sont réguliers mais qu'il arrive qu'ils soient modifiés à cause de circonstances particulières (éléments de perturbations).

A partir d'entrevues effectuées avec une cinquantaine de mères, Lautrey a construit un questionnaire visant à mesurer le type de structuration éducative parentale. Pour chacune des questions, les parents doivent choisir parmi trois possibilités, celle qui correspond le plus à la réalité familiale, chaque choix de réponse correspondant à un des trois types de structuration (faible 
$(f)$, souple (s), rigide (r)). Il classe ensuite chaque famille selon le nombre de réponses de chaque type et obtient six groupes:

A) f $>$ r $>$ s; B) f $>$ s $>$ r; C) s $>$ f $>$ r; D) $s>$ r $>$ f; E) r $>$ s $>$ f; F) r $>$ f $>$ s.

Une recherche d'envergure a permis à Lautrey de vérifier son hypothèse. Il a administré le questionnaire à 1385 familles et a ensuite évalué les garçons (qui avaient 10 ans) à l'aide de deux tests d'intelligence générale: l'épreuve d'appréhension de relations/cm2 (AR/CM2) et l'épreuve collective pour enfants de 11 ans (EC/11). Selon l'hypothèse de Lautrey, les enfants provenant des familles différentes quant à leur structuration éducative devraient présenter des résultats différents aux tests d'intelligence, ceux des familles $C$ et $D$ étant les meilleurs et ceux des familles $B$ et $E$ étant supérieurs à ceux des familles $\mathrm{A}$ et $\mathrm{F}$. Le Tableau 1 présente schématiquement les résultats attendus, selon l'hypothèse de Lautrey, ainsi que les résultats obtenus selon les tests utilisés.

Tableau 1

Résultats théoriquement prévus dans l'hypothèse et résultats obtenus par Lautrey

$$
\begin{array}{ll}
\text { Hypothèse } & \mathrm{A}<\mathrm{B}<\mathrm{C} ? \mathrm{D}>\mathrm{E}>\mathrm{F}^{5} \\
\mathrm{AR} / \mathrm{CM} 2 & \mathrm{~A}<\mathrm{B}=\mathrm{C}>\mathrm{D}>\mathrm{E}=\mathrm{F} \\
\mathrm{EC} / 11 & \mathrm{~A}<\mathrm{B}=\mathrm{C}>\mathrm{D}>\mathrm{E}=\mathrm{F}
\end{array}
$$

On peut constater qu'à chaque fois que les résultats trouvés ne vont pas dans le sens de l'hypothèse, ils correspondent à une indifférenciation entre le nombre de réponses souples et le nombre de réponses faibles. Par exemple, les groupes $\mathrm{E}$ et $\mathrm{F}$ se retrouvent à égalité. Les parents de ces deux groupes ont en commun le fait qu'ils donnent plus de réponses de type rigide alors que ce qui les distingue c'est le nombre de réponses faibles et souples. Il est à noter que ces résultats correspondent à ceux obtenus après avoir retiré de l'échantillon les enfants n'ayant pas la nationalité française. Avant qu'ils ne soient retirés, les résultats à l'AR/CM2 étaient conformes à l'hypothèse.

\footnotetext{
${ }^{5}$ On ne peut, selon la théorie présentée, savoir quel type de structuration est plus favorable entre les types C et $D$. Les deux types étant caractérisés par un nombre de réponses souples plus élevé, ce qui les différencie est le nombre de réponses de types rigide et faible
} 
Les attitudes et pratiques éducatives parentales varient d'une culture à une autre. Aussi, il devient intéressant de se questionner sur l'effet de la structuration éducative parentale sur le développement cognitif dans un contexte culturel différent. De plus, tel que le suggère Storfer (1990), certaines attitudes et pratiques éducatives parentales qui s'avèrent favorables au développement intellectuel des enfants à un moment donné de son développement ne le sont pas nécessairement à toutes les étapes de son développement. Storfer remarque également que les attitudes et conduites éducatives des parents se modifient à mesure que les enfants grandissent.

Dans la présente recherche nous tenterons donc de vérifier l'impact du type de structuration éducative parentale sur le développement cognitif d'enfants québécois de 5 ans. Il nous semble que l'enfant de 5 ans, présentant un fonctionnement cognitif pré-opératoire, sera d'autant plus sensible à la présence de régularités dans l'environnement que l'enfant plus vieux caractérisé par sa capacité opératoire. De plus, l'enfant de 5 ans est caractérisé par une scolarité absente ou très récente 6 .

Notre recherche est également caractérisée par le fait que nous considérons, contrairement aux travaux de Lautrey ainsi qu'à une majorité de recherches, les attitudes et pratiques éducatives aussi bien des mères que des pères.

Reste à résoudre le problème du choix d'une mesure du développement cognitif. Il nous semble important que celle-ci soit conforme à la théorie piagétienne qui est à la base du concept de structuration éducative parentale de Lautrey. Les épreuves piagétiennes classiques sont évidemment inappropriées pour des enfants de stade pré-opératoire. Les travaux d'OrsiniBouichou (1982) portant sur le fonctionnement cognitif des enfants de stade pré-opératoire, offrent les éléments de solution à notre problème.

Orsini-Bouichou s'est en effet intéressée à l'intelligence de l'enfant pré-opératoire. Soucieuse de laisser l'intelligence de l'enfant s'exprimer librement, elle a imaginé une situation (épreuves de libres combinaisons d'objets) qui offrirait à l'enfant une liberté d'action à l'intérieur d'un cadre comportant le moins de contraintes (consignes) possible. Elle a pu constater que des règles interviennent dans l'activité spontanée des enfants de stade pré-opératoire. Elle a appelé régularité

6 Seulement des classes maternelles pour les enfants de 5 ans sont offertes à l'ensemble de la population québécoise. 
un ensemble de comportements exprimant une même règle. Ces régularités diffèrent qualitativement d'un âge à un autre. Ainsi, des régularités plus complexes apparaissent à mesure que l'enfant grandit.

Palacio-Quintin (1988), reprenant des expériences avec ces épreuves de libres combinaisons d'objets $^{7}$, a pu confirmer la présence des régularités telles que décrites par Orsini-Bouichou mais a également identifié quelques types nouveaux. De plus, accordant une valeur à chaque type de régularité selon son degré de complexité, ainsi qu'à la variété de production, elle a pu quantifier les résultats et ainsi déterminer un indice de développement. Ensuite, Palacio-Quintin (1990) a pu démontrer, dans une étude longitudinale, que les enfants qui, à 4 ans, présentaient un indice de développement plus élevé étaient ceux qui avaient le plus de chances d'avoir acquis la capacité de conservation à 7 ans. Cet indice, donc, ne correspond pas à une mesure statique du niveau de développement tel qu'il se présente à un moment donné, mais permet davantage une investigation du fonctionnement cognitif d'un point de vue dynamique.

Dans cette recherche, nous tenterons donc de démontrer que les jeunes enfants provenant de milieux familiaux à structuration éducative souple présenteront un meilleur fonctionnement cognitif que les enfants provenant des milieux à structuration aléatoire (faible) ou rigide.

Nous faisons donc l'hypothèse qu'il y aura une relation positive entre l'indice de développement cognitif de l'enfant et la structuration éducative parentale souple et une absence de relation ou une relation négative entre l'indice de développement et les structurations éducatives faible et rigide.

\section{METHODOLOGIE}

\section{Echantillon}

L'échantillon est composé des 47 familles (27 garçons et 20 filles) qui ont été retenues à partir d'un échantillon original de 60 familles. Les enfants sont âgés de 60 à 76 mois. Le Tableau 2 présente l'âge moyen (et écart-type) des garçons et des filles.

7 Ces épreuves, utilisées dans le cadre de la présente recherche, seront présentées de façon plus explicite dans la partie consacrée à la méthodologie. 


\section{Tableau 2}

Age moyen (écart-type et dispersion) des garçons et des filles

\begin{tabular}{|l|c|c|c|}
\hline & garçons $(\mathrm{N}=27)$ & filles $(\mathrm{N}=20)$ & garçons et fillles \\
\hline âge moyen & 69,37 & 70,6 & 69,89 \\
écart-type & 4,03 & 4,11 & 4,07 \\
dispersion & $60-75$ & $63-76$ & $60-76$ \\
\hline
\end{tabular}

De façon à contrôler les effets attribuables à des différences culturelles, ces familles sont toutes d'origine québécoise francophone et résidant dans la région de Trois-Rivières. De plus, différents chercheurs ayant montré qu'il existe des différences entre les familles bi-parentales, mono-parentales et reconstituées quant aux attitudes et pratiques éducatives parentales et leurs influences sur le développement des enfants, nous avons restreint notre étude, afin d'éliminer l'influence de cette variable, aux familles dont l'enfant vit avec ses deux parents biologiques. Enfin, nous avons choisi notre échantillon, au hasard, à l'intérieur d'une diversité de quartiers de la ville, afin que celui-ci soit le plus représentatif possible de la population générale en ce qui concerne le statut socio-économique.

\section{Instruments utilisés}

A - Le type de structuration éducative parentale.

Le type de structuration éducative parentale a été mesuré à l'aide du Questionnaire sur la structuration éducative parentale (QSEP) de Palacio-Quintin et Lavoie (1986). Le QSEP est une adaptation du questionnaire que Lautrey (1980) avait construit à l'intention de familles françaises ayant un enfant de 10 ans. Le QSEP a été conçu de façon à répondre à la réalité culturelle québécoise et aux caractéristiques d'enfants d'âge pré-scolaire. Il comporte 17 items pour lesquels les parents doivent choisir, parmi les trois réponses possibles, celle qui correspond le mieux à leurs comportements et habitudes quotidiennes. Le type de structuration éducative est déterminé par le nombre de réponses faibles, souples et rigides. 
B - Le niveau de développement cognitif

Le niveau de développement cognitif des enfants est évalué à l'aide de l'épreuve de libres combinaisons d'objets (adaptation des épreuves d'Orsini-Bouichou par Palacio-Quintin, 1983).

\section{Matériel:}

- une boîte de bois, en forme de plumier, divisée en 24 cases et munie d'un couvercle amovible qui peut glisser le long du plumier. Une fenêtre à l'extrémité du couvercle permet de voir à l'intérieur de chacune des cases à mesure que l'on glisse le couvercle, cachant ainsi les cases précédentes;

- une boîte contenant 25 perles rouges et 25 perles blanches mélangées.

\section{Déroulement de l'épreuve:}

L'expérimentateur présente le matériel à l'enfant. Il lui apprend comment on peut utiliser la boîte et son couvercle. Il lui présente ensuite les perles sans mentionner qu'il s'agit de billes de couleurs différentes.

Il demande ensuite à l'enfant de mettre une bille dans chaque case (et seulement une) en utilisant la fenêtre et ce, en choisissant comme il (l'enfant) le veut.

L'expérimentateur note la mise de l'enfant sur un protocole. Une fois le plumier rempli, l'expérimentateur demande à l'enfant d'expliquer la stratégie qu'il a utilisée ("Qu'est-ce que tu viens de faire?", "Explique comment tu as fait"). L'expérimentateur vide ensuite le plumier et demande à l'enfant de recommencer en faisant quelque chose de différent, quelque chose qu'il n'a pas encore fait, et reprend la procédure suivie précédemment. On répète ainsi l'expérience de façon à ce que l'enfant fasse 8 essais (production et verbalisation).

\section{Cotation:}

Nous avons utilisé la cotation suggérée par Palacio-Quintin (1988). Les divers types de régularités qu'elle a identifiés sont les suivants: uniformité (U), uniformité coupée (UC), alternance symétrique élémentaire (ASE), deux parties (Par), alternance de pluralités (P/P), altemance d'unités 
et de pluralités (U/P), famille d'alternances symétriques (ASF), alternance complexe (AC). Une valeur est accordée à chaque type de régularités selon son niveau de complexité. Dans le cas d'une mise composée de deux types de régularités différents (mixte ou M), la valeur accordée est celle de la régularité la plus complexe à laquelle on ajoute 0,5 . Le Tableau 3 présente les valeurs correspondant à chaque type de régularités.

\section{Tableau 3}

Valeur accordée pour chaque type de régularités

\begin{tabular}{|l|l|}
\hline régularité & score \\
\hline $\mathrm{U}$ & 1 \\
$\mathrm{UC}, \mathrm{PAR}, \mathrm{P} / \mathrm{P}$ & 1,5 \\
$\mathrm{ASE}$ & 2 \\
$\mathrm{U} / \mathrm{P}$ & 2,5 \\
$\mathrm{ASF}$ & 3 \\
$\mathrm{AC}$ & 4 \\
\hline
\end{tabular}

Pour l'ensemble des 8 essais, on note le nombre de régularités différentes produites par l'enfant, ce qui nous donne l'indice de variabilité (IVA). L'indice de développement (ID) est obtenu en faisant la somme des valeurs correspondant à chaque régularité produite par l'enfant et en le multipliant par l'indice de variabilité $\left(\mathrm{D}=\sum\right.$ scores $\mathrm{X}$ IVA).

\section{L'expérience}

60 familles ont d'abord été sélectionnées (selon les critères énumérés plus haut) et rencontrées afin de compléter le QSEP. Les mères et les pères de chaque famille furent rencontrés à leur domicile par l'expérimentateur. Celui-ci leur présentait le questionnaire et précisait aux parents qu'ils devaient choisir leurs réponses en fonction de leurs habitudes familiales et sans tenir compte d'un idéal qu'ils aimeraient atteindre. Chaque parent a rempli le questionnaire individuellement et l'expérimentateur demeurait disponible afin d'aider les parents qui pouvaient bénéficier de son support pour la compréhension des questions. 
Afin de mesurer l'impact de la structuration éducative parentale sur le développement de l'enfant, chaque enfant a été évalué 14 mois ( $+/$ - 1 mois) après que ses parents eurent complété le QSEP. 47 enfants sur les 60 qui composaient l'échantillon original ont alors pu être évalués. Les 13 autres n'ont pu l'être, soit parce que les parents ont alors refusé, soit parce qu'il nous a été impossible de les rejoindre (déménagements). L'expérimentateur a rencontré les enfants individuellement afin de leur faire passer les épreuves de libres combinaisons d'objets selon la procédure présentée plus haut.

Les recherches effectuées à l'aide du QSEP montrent que la distribution des réponses données par les familles québécoises à ce questionnaire est caractérisée par une incidence beaucoup plus forte de réponses souples que de réponses faibles ou rigides (Coderre, 1990; Palacio-Quintin et Lacharité, 1989). Dans ces circonstances, la méthode d'analyse statistique qui nous semble la plus appropriée afin de mesurer la relation entre la structuration éducative parentale et le fonctionnement cognitif est celle qui consiste à vérifier la corrélation entre le nombre de réponses de chaque type (faible, souple, rigide) et l'indice de développement.

\section{RESULTATS}

La présentation des résultats se fera en trois étapes. Nous présenterons d'abord les résultats relatifs aux performances des enfants aux épreuves de libres combinaisons d'objets. Nous verrons ensuite les caractéristiques de notre échantillon en ce qui concerne la structuration éducative et, enfin, nous présenterons les corrélations qui existent entre les deux mesures.

\section{Epreuves de libres combinaisons d'objets}

Sur l'ensemble de la production des enfants à ces épreuves, on note que $57,7 \%$ de leurs mises sont faites au hasard alors que $42,3 \%$ sont organisées sous forme d'une régularité. La Figure 1 présente la fréquence des régularités de chaque type produites par les sujets. 
Figure 1

Fréquence des régularités de chaque type produites par les sujets

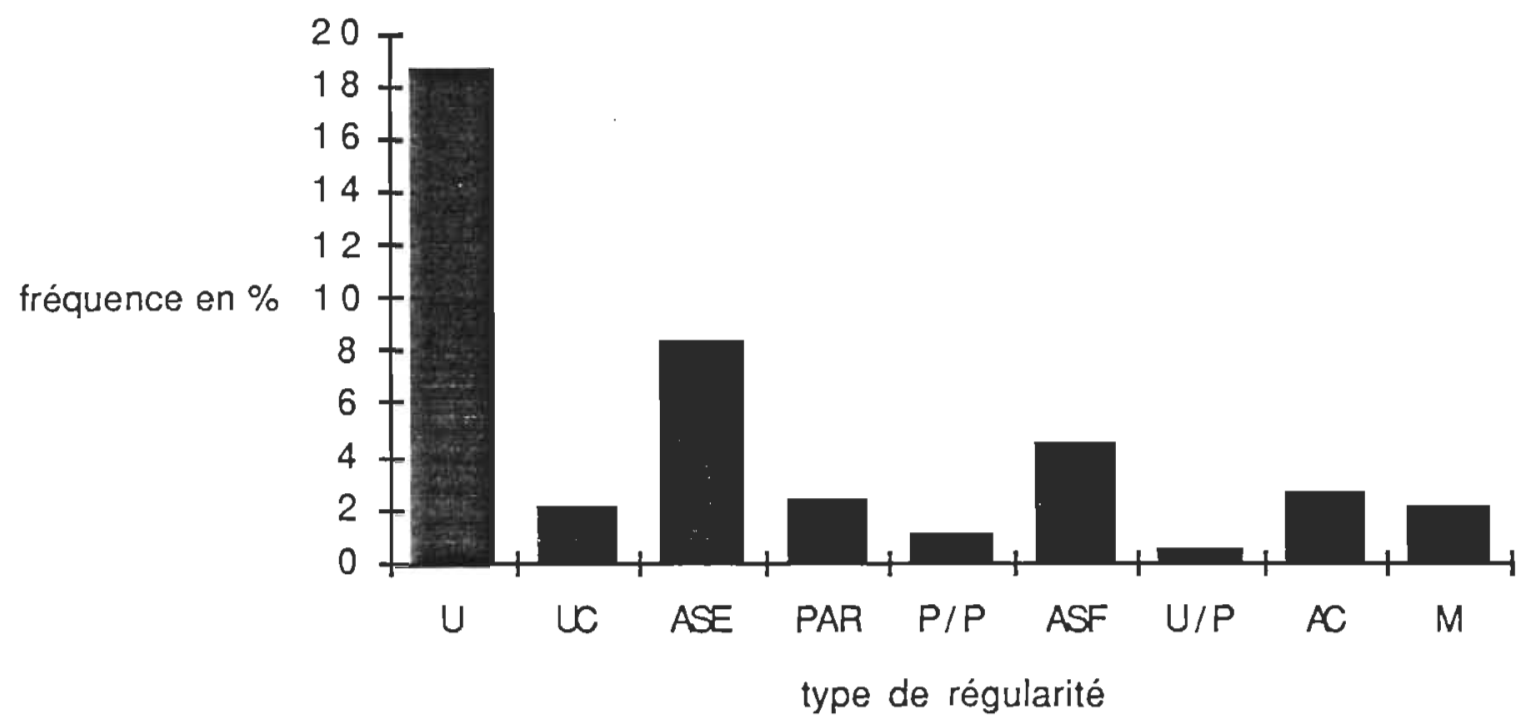

Le Tableau 4 présente le score moyen à l'indice de développement

Tableau 4

Moyenne, écart-type et dispersion des résultats à lindice de développement

\begin{tabular}{|l|c|c|c|c|}
\hline \multicolumn{1}{|c|}{ dispersion } & Noyenne & écart type & \\
\hline $\begin{array}{l}\text { Indice de } \\
\text { développement }\end{array}$ & $47^{*}$ & 31.45 & 49.07 & $0-232$ \\
\hline
\end{tabular}

* Il est à noter que 12 de ces sujets (25.5\%) ont obtenu 0 à l'indice de développement.

\section{Structuration éducative parentale}

La Figure 2 présente la distribution du nombre de réponses de chaque type telles que données par les parents au QSEP alors que la Figure 3 présente la distribution des mères et des pères selon le type de structuration éducative. 
Figure 2

Distribution des réponses de chaque type telles que données par les mères et les pères au QSEP

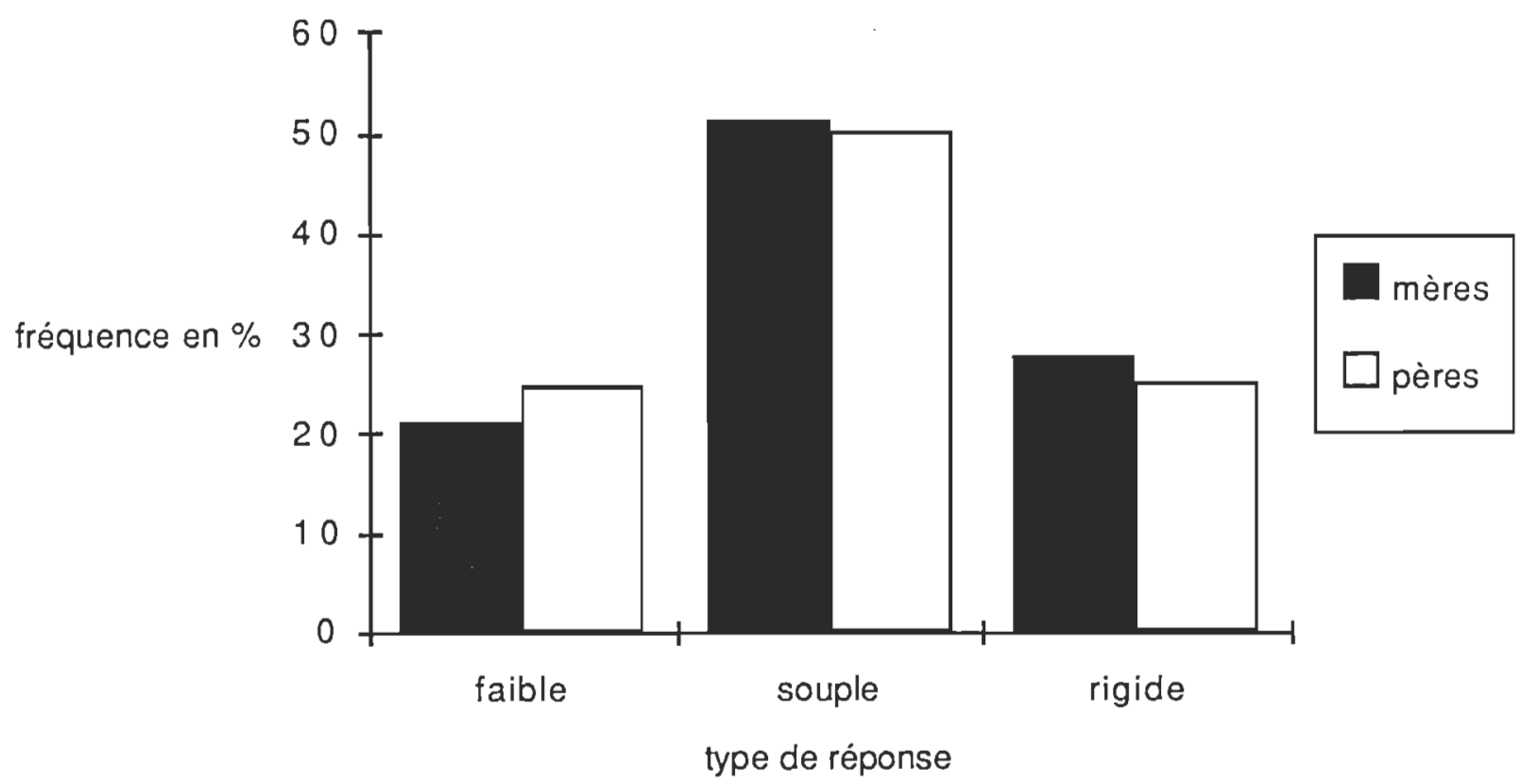

Figure 3

Distribution des familles selon le type de structuration

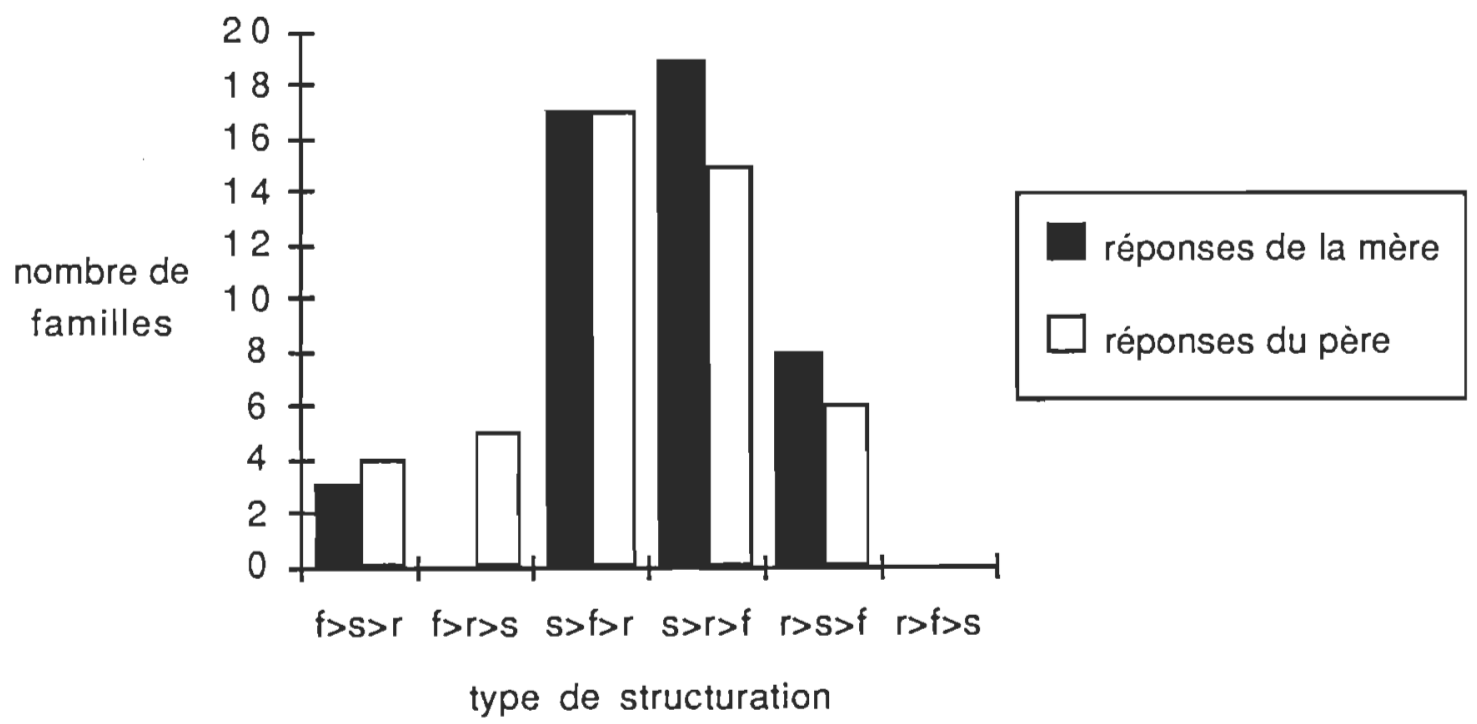


La Figure 2 nous permet de constater la prépondérance du nombre de réponses souples sur le nombre de réponses rigides ou faibles. Par conséquent, notre échantillon est composé d'une majorité de familles à structuration souple (voir Figure 3). On remarque également une certaine similarité entre la distribution des réponses des mères et celle des réponses des pères. Les corrélations entre le nombre de réponses de chaque type données par les couples parentaux sont toutes significatives. La corrélation entre le nombre de réponses faibles des pères et des mères est de $.62(\mathrm{p}<.001)$, celle entre le nombre de réponses souples est de $.44(\mathrm{p}=.002)$ et, enfin, le nombre de réponses rigides des pères et des mères présentent une corrélation de $.49(\mathrm{p}=.001)$. Notons enfin l'absence, dans notre échantillon, de mères et de pères présentant une structuration de type "r>f>s".

La relation entre la structuration éducative parentale et le développement cognitif de l'enfant

Le Tableau 5 présente les corrélations entre le nombre de réponses faibles, souples et rigides au QSEP et l'indice de développement. Etant donné l'aspect développemental des épreuves de libres combinaisons d'objets et la dispersion de nos sujets quant à l'âge (16 mois), nous avons inséré, lors de l'analyse statistique, la variable âge comme variable de contrôle. De plus, nous avons retiré de l'échantillon les 12 sujets qui ont obtenu 0 à l'indice de développement.

Tableau 5

Corrélations partielles (l'effet de l'âge étant contrôlé) entre les résultats obtenus à l'indice de développement et le nombre de réponses faibles, souples et rigides données par chacun des 2 parents

\begin{tabular}{|l|lll|lll|}
\hline & \multicolumn{2}{|c|}{ réponses de la mère } & \multicolumn{3}{c|}{ réponses du père } \\
& faible & souple & rigide & faible & souple & rigide \\
\hline Indice & -.1616 & .0945 & .0824 &.-3655 & .2405 & .1300 \\
& $(\mathrm{p}=.181)$ & $(\mathrm{p}=.297)$ & $(\mathrm{p}=.322)$ & $(\mathrm{p}=.017)$ & $(\mathrm{p}=.085)$ & $(\mathrm{p}=.232)$ \\
\hline
\end{tabular}

A la lumière de ces résultats, nous ferons trois constatations: 1) les corrélations entre les réponses faibles des mères et des pères et l'indice de développement sont négatives, quoiqu'elles ne 
soient pas significatives dans le cas des mères. En effet, seule la corrélation entre le nombre de réponses faibles du père et l'indice de développement est significative; 2) Bien que les corrélations ne soient pas significatives, l'indice de développement a tendance à corréler davantage avec le nombre de réponses souples que le nombre de réponses rigides; 3 ) les corrélations, qu'elles soient négatives ou positives, sont en général plus importantes lorsqu'on considère les réponses du père que celles de la mère.

En résumé, malgré le fait que seule une des six corrélations est significative, l'ensemble de ces comélations tendent à nous montrer que la structuration faible a un impact négatif sur le développement de l'enfant, alors que la structuration souple a un impact positif plus important que la structuration rigide.

\section{CONCLUSION}

Nos résultats montrent que la structuration éducative faible chez les pères, semble moins favorable au développement cognitif des enfants que la structuration souple ou rigide. Cette constatation diffère de celle de Lautrey (1980). En effet, les résultats de Lautrey - issus d'une recherche qu'il a mené avec des enfants de 10 ans - montrent une différence plus nette entre les milieux rigides et souples, en faveur de ces derniers, alors que les milieux souples et faibles se présentent comme étant moins différenciés.

Il semble donc qu'un minimum de structure au niveau des habitudes et règles éducatives minimum qui est présent autant dans les milieux à structuration souple que dans les milieux à structuration rigide - se révèle plus nécessaire au bon développement du jeune enfant .

Cependant, les corrélations observées ne mettent pas en évidence de façon très nette la supériorité de l'attitude souple sur l'attitude rigide quant à son effet sur le fonctionnement cognitif. Tout au moins, cette supériorité n'est pas mise en évidence par le type d'épreuves utilisées ici.

Lautrey $(1978,1980)$ remarque en effet une différence entre les enfants des milieux souples et rigides quant à leurs niveaux structuraux et leurs niveaux de fonctionnement. Il trouve cependant ces différences chez des enfants plus âgés, se situant au moins au niveau des opérations concrètes. 
Nos résultats semblent plutôt indiquer que le fonctionnement cognitif de l'enfant de 5 ans serait davantage sensible à une trop grande absence de régularités des événements. Ainsi, une fois un minimum de structure atteint, les milieux fortement structurés et les milieux plus souples présentent peu de différences quant à la répercussion qu'ils ont, à court terme, sur l'intelligence de l'enfant dans ses aspects fonctionnels.

Il est nécessaire, cependant, de considérer ces résultats avec prudence, étant donné que les types de structuration ne se distribuent pas uniformément, le nombre de réponses souples étant fortements majoritaires.

Nous avons considéré, lors de nos analyses, les réponses du père et celles de la mère de façon séparée. Par rapport aux recherches antérieures, il s'agit de données nouvelles. Cependant, une analyse de l'effet d'interaction entre les deux permettrait d'approfondir la compréhension que nous avons de la relation entre structuration éducative parentale et développement cognitif. A ce sujet, nous avons démontré, dans la partie consacrée à la présentation des résultats, qu'il existe une corrélation entre le nombre de réponses de chaque type des mères et des pères. Cependant, si nous classons les parents selon les six catégories utilisées par Lautrey (1980), nous constatons que pour environ la moitié des couples (23 sur 47 ) les deux parents ne présentent pas le même type de structuration éducative.

Afin d'analyser l'effet d'interaction de la structuration éducative des deux parents, nous ne pensons pas qu'une méthode adéquate consisterait en une simple addition des réponses maternelles et paternelles. On risquerait ainsi d'apparier des familles dont le nombre de réponses cumulées (mère + père) est le même mais dont des différences importantes existent si l'on considère l'un et l'autre des deux parents séparément. Quant à l'idée de pondérer les types de réponses, elle nous paraît tout aussi infructueuse. En effet, rien ne nous indique quel poids donner à une réponse souple par rapport à une réponse faible ou rigide.

Par contre, une démarche qui consisterait à faire un inventaire de patterns de réponses possibles, tout en tenant compte des réponses de la mère et du père, permettrait ensuite de classer les familles à l'étude selon le type de structuration sans isoler chacun des deux parents. Evidemment, vu le nombre considérable de patterns possibles, une recherche utilisant une telle 
méthodologie nécessiterait un échantillon d'une taille beaucoup plus importante que celui dont nous disposons actuellement. 


\section{REFERENCES}

Baker, C. T., Nelson, V. L. \& Sontag, L. W. (1958). Mental growth and personality development: A longitudinal study. Monographs of the Society for research in child development, $23, n^{\circ} 68$.

Baldwin, A. L., Kalhorn, J. \& Breese, F. H. (1945). The appraisal of parent behavior. Psychological Monographs, 58, whole $\mathrm{n}^{\circ} 268$.

Banner, C. N. (1979). Child-rearing attitudes of mothers of under-, average-, and over-achieving children. British Journal of Educational Psychology, 49, 150-155.

Bayley, N. (1933). Mental growth during the first three years: A developmental study of 61 children by repeated tests. Genetic Psychological Monographs, 14, 1-92.

Bayley, N. \& Schaefer, E. S. (1964). Correlations of maternal and child behaviors with the development of mental abilities: Data from the Berkeley Growth Study. Monographs of the Society for Research in Child Development, 29, whole n ${ }^{\circ} 97$.

Becker, W. C., Peterson, D. R., Hellmer, L. A., Shoemaker, D. J. \& Quay, H. C. (1959). Factors in parental behavior and personality as related to problem behavior in children. Journal of Consulting Psychology, 23, 107-118.

Bee, H. L., Barnard, K. E., Eyres, S. J., Gray, C. A., Hammond, M. A., Spietz, A. L., Snyder, C. \& Clark, B. (1982). Prediction of IQ and language skill from perinatal status, child performance, family characteristics, and mother-infant interaction. Child Development, 53, $1134-1156$.

Bing, E. (1963). Effect of childrearing practices on development of differential cognitive abilities. Child Development, 34, 631-648.

Bradley, R. H. \& Caldwell, B. M. (1976a). Early home environment and changes in mental test performance in children 6 to 36 months. Developmental Psychology, 12, 93-97.

Bradley, R. H. \& Caldwell, B. M. (1976b). The relation of infants' home environments to mental test performance at fifty-four months: A follow-up study. Child Development, 47, 1172-1174.

Bradley, R. H. \& Caldwell, B. M. (1977). Home Observation for Measurement of the Environment: A validation study of screening efficiency. American Journal of Mental Deficiency, 81, 417-420.

Bradley, R. H. \& Caldwell, B. M. (1978). Screening the environment. American Journal of Orthopsychiatry, 48, 114-130.

Busse, T. V. (1969). Child-rearing antecedents of flexible thinking. Developmental Psychology, 1 , 585-591.

Caldwell, B., Heider, J. \& Kaplan, B. (1966). The inventory of home stimulation. Paper presented at the annual meeting of the American Psychological Association, Washington, DC 
Caldwell, B. \& Richmond, J. (1967). Social class level and the stimulation potential of the home. The Exceptional Infant, 1, 453-466.

Coderre, R. (1990). L'influence de la structuration éducative parentale sur les enfants de niveau préscolaire. Mémoire de maîtrise, UQTR.

Cross, H. J. (1966). The relation of parental training conditions to conceptual level in adolescent boys. Journal of Personality, $\underline{34}, 348-365$.

Drews, E. M. \& Teahan, J. E. (1957). Parental attitudes and academic achievement. Journal of Clinical Psychology, $13,328-332$.

Elardo, R., Bradley, R. \& Caldwell, B. M. (1975). The relation of infants' home environments to mental test performance from six to thirty-six months: A longitudinal analysis. $\underline{\text { Child }}$ Development, 46, 71-76.

Golden, M., Birns, B., Bridger, W \& Moss, A. (1971). Social-class differentiation in cognitive development among black preschool children. Child Development, 42, 37-45.

Goldschmid, M. L. (1968). The relation of conservation to emotional and environmental aspects of development. Child Development, 38, 579-589.

Goldstein, D., Meyer, W. J. \& Egeland, B. (1978). Cognitive performance and competence characteristics of lower- and middle-class preschool children. The Journal of Genetic Psychology, 132, 177-183.

Gottfried, A. W. (1984). Home environment and early cognitive development. Longitudinal research. Orlando, Fla: Academic Press.

Hamilton, V. (1971). Effect of maternal attitude on development of logical operations. Perceptual and Motor Skills, 33, 63-69.

Hilliard, T. \& Roth, R. M. (1969). Maternal attitudes and the non-achievement syndrome. Personnel and Guidance Journal, 47, 424-428.

Hurley, J. R. (1959). Maternal attitudes and children's intelligence. Joumal of Clinical Psychology, 15, 291-292.

Kent, N. \& Davis, D. R. (1957). Discipline in the home and intellectual development. British Joumal of Medical Psychology, 30, 27-33.

Lautrey, J. (1978). Structuration de l'environnement familial et développement cognitif. cahiers de psychologie 21, 99-110.

Lautrey, J. (1980). Classe sociale, milieu familial, intelligence. Paris: Presses Universitaires de France.

Moss, H. A. \& Kagan, J. (1958). Maternal influences on early IQ scores. Psychological reports, 4, 655-661.

Nichols, R. C. (1964). Parental attitudes of mothers of intelligent adolescents and creativity of their children. Child Development, 35, 1041-1049. 
Orsini-Bouichou, F. (1982). L'intelligence de l'enfant: Ontogénèse des invariants. Paris: Centre National de la Recherche Scientifique.

Palacio-Quintin, E. (1983). Adaptation des épreuves de Orsini-Bouichou. Document polycopié. GREDE, UQTR.

Palacio-Quintin, E. (1988). The relation between cognitive functioning and operational structures. A longitudinal study of four to seven-year old children. communication presentee au Child Development Congress, Waterloo.

Palacio-Quintin, E. \& Lacharité, C. (1989). Variables de l'environnement familial qui affectent le développement intellectuel des enfants de milieu socio-économique faible. Rapport de recherche. GREDE, UQTR.

Palacio-Quintin, E. (1990). Links between functional and structural aspects of thinking. Communication présenté au $20^{\text {th }}$ Anniversary Symposium Jean Piaget Society, Philadelphie, 31 mai au 2 juin.

Palacio-Quintin, E. \& Jourdan-Ionescu, C. (1991). Les enfants de quatre ans: La mesure du Home et du QI en fonction du niveau socio-économique et culturel. Enfance, 45, 99-110.

Palacio-Quintin, E. \& Lavoie, T. (1986). Questionnaire sur la structuration éducative parentale. Version préscolaire (QSEP). Document polycopié, GREDE, UQTR.

Radin, N. (1974). Observed maternal behavior with four year old boys and girls in lower-class families. Child Development, 45, 1126-1131.

Scarr, S. (1981). Race social class, and individual differences in IQ. Hillsdale, NJ: Lawrence Erlbaum.

Schaefer, E. S. (1959). A circumplex model for maternal behavior. Journal of Abnormal and Social Psychology, 59, 226-235.

Storfer, M. D. (1990). Intelligence and giftedness. The contributions of heredity and early environment. San Francisco: Jossey-Bass.

Wachs, T. D. (1978). The relationship of infants' physical environment to their Binet performance at 2 1/2 years. International Journal of Behavioral Development, 1, 51-65.

Wachs, T. D. (1979). Proximal experience and early cognitive-intellectual development: The physical environment. Merrill-Palmer Quarterly, 25, 3-41.

Wulbert, M., Inglis, S., Kriegsmann, E. \& Mills, B. (1975). Language delay and associated mother-child interactions. Developmental psychology, 11, 61-70. 
Résumé

Un grand nombre de recherches ont tenté de vérifier la relation entre attitudes parentales et développement intellectuel des enfants. Parmi les approches utilisées, celle de Lautrey (1980) se distingue par le fait qu'elle s'inspire de la théorie du développement cognitif de Piaget. La présente recherche, qui va dans le même sens, a pour but de vérifier la relation entre le type de structuration éducative parentale et le fonctionnement cognitif d'enfants québécois de 5 ans. La structuration éducative parentale est mesurée avec le QSEP (Palacio-Quintin et Lavoie 1986). Le fonctionnement cognitif est mesuré à l'aide des épreuves de libres combinaisons d'objets élaborées par OrsiniBouichou (1982). Les résultats, obtenus à partir d'un échantillon de 47 familles, indiquent qu'une faible structuration éducative, autant chez les mères que chez les pères, est reliée à un moins bon fonctionnement cognitif que la structuration éducative parentale souple ou rigide.

\begin{abstract}
Many studies have attempted to verify the relationship between parental attitudes and children's intellectual development. Lautrey's method differs from all: it is based on the Piaget's theory about cognitive development (Lautrey, 1980). This study which is inspired of Lautrey's one is designed to examine the relationship between parental educative structuration and cognitive functioning of 5 years old quebecois children. QSEP (Palacio-Quintin et Lavoie, 1986) is used to measure the parental educative structuration. Cognitive functioning is measured with Orsini-Bouichou's free combinations of objects task (Orsini-Bouichou, 1982). Results gained from a sample of 47 families show that a low parental educative structuration is related with a lower level of cognitive functioning than the flexible of rigid parental educative structuration. This finding concerns mothers alike fathers.
\end{abstract}




\section{REMERCIEMENTS}

L'auteur désire exprimer toute sa reconnaissance à sa directrice de recherche, madame Ercilia Palacio-Quintin, Ph. D., professeure au département de Psychologie, pour son support constant et sa précieuse collaboration tout au long de cette recherche. 